\title{
A HELIUM FILM COATED QUASI-PARABOLIC MIRROR TO FOCUS A BEAM OF ULTRA-COLD SPIN POLARIZED ATOMIC HYDROGEN
}

\author{
V.G. Luppov*, W.A. Kaufman, K.M. Hill, R.S. Raymond, A.D. Krisch \\ Randall Laboratory, University of Michigan, Ann Arbor, MI 48109-1120 USA
}

\section{ABSTRACT}

A $350 \mathrm{mK}$ helium-4-coated mirror was used to increase the intensity of an ultra-cold electron-spin-polarized atomic hydrogen beam. The mirror uses the observed specular reflection of atomic hydrogen from a superfluid-helium-covered surface $^{1}$. A quasi-parabolic polished copper mirror was installed with its focus at the $5 \mathrm{~mm}$ diameter exit aperture of an atomic hydrogen stabilization cell in the gradient of an $8 \mathrm{~T}$ solenoid field. The four-coned mirror shape, which was designed specifically for operation in the gradient, increased the beam intensity focused by a sextupole magnet into a compression tube detector by a factor of about 7.5.

Using the Michigan prototype jet ${ }^{2}$ we investigate a relatively new ultra-cold method which uses a temperature about $0.3 \mathrm{~K}$ and a magnetic field above $5 \mathrm{~T}$ to produce a high density electron-spin-polarized atomic hydrogen beam. Depolarization and recombination into molecular hydrogen are strongly suppressed because the average thermal energy is much too small to flip the electron spin. The method, called "no microwave" extraction, uses the sharp gradient of a strong magnetic field to separate the cold hydrogen atoms of different electron spin states.

The Michigan prototype jet using the "no-microwave" extraction is shown in Fig. 1.

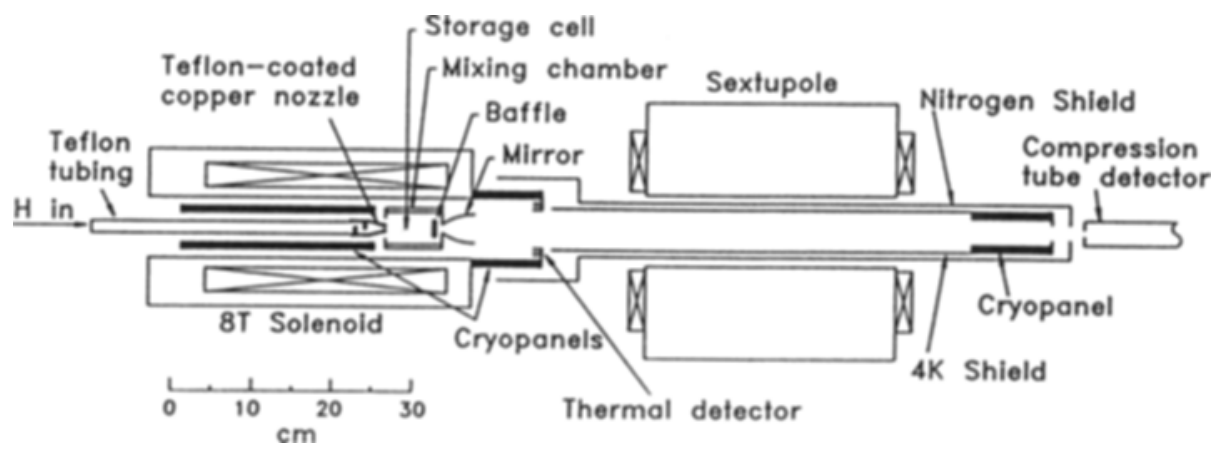

FIG. 1. Schematic diagram of the Michigan prototype ultra-cold spin-polarized atomic hydrogen jet. 
The atomic hydrogen was produced in a room temperature rf dissociator and guided to an ultra-cold stabilization cell through a teflon tube with a teflon coated copper nozzle held at about $20 \mathrm{~K}$. The double walls of the cell formed the horizontal mixing chamber of the dilution refrigerator; its cooling power was about $25 \mathrm{~mW}$ at $350 \mathrm{mK}$. The cell's entrance and exit apertures were respectively located at $95 \%$ and $65 \%$ of the central field of the $8 \mathrm{~T}$ superconducting solenoid. The cell typically operated at a temperature of $350 \mathrm{mK}$, and was completely covered with a superfluid ${ }^{4} \mathrm{He}$ film to suppress the surface recombination of hydrogen atoms.

After the hydrogen atoms were sufficiently thermalized by collisions with the cell surfaces, the magnetic field gradient physically separated the atoms according to their two different electron-spin states. The atoms in the two lowest hyperfine states (high field seekers) were attracted toward the high field region. Most of these atoms eventually escaped from the cell through the $50 \mathrm{~mm}^{2}$ annular gap around the entrance nozzle. These atoms then recombined on bare surfaces; the resulting molecular hydrogen was pumped away by cryopanels and other cold surfaces. The atoms in the two higher energy hyperfine states (low field seekers) were repelled toward the low field regions, where they collected and then effussed from the $5 \mathrm{~mm}$ diameter exit aperture. After emerging from the exit aperture, the electron-spin-polarized atoms were magnetically accelerated by the remaining field gradient.

We measured the extracted atomic hydrogen beam flux, using a compression tube (CT) detector mounted downstream of both the cell and the sextupole magnet. A tracking simulation for our prototype jet with a 7.3 T solenoid field indicates that typically $1 \%$ of the beam effussing from our ultra-cold cell can be focused into the CT by our sextupole. Increasing either the sextupole aperture or the solenoid field would improve the beam transport, but requires a serious modification of the apparatus.

The quantum reflection of cold hydrogen atoms from a helium-film-covered surface below $0.5 \mathrm{~K}$ was demonstrated by Berkhout et al. ${ }^{1}$ They measured about $80 \%$ specular reflectivity for normal incidence on a hemispherical optical quality concave quartz mirror coated with a $100 \mathrm{mK}$ saturated ${ }^{4} \mathrm{He}$ film.

We designed a "parabolic" mirror to use the specular reflection as an "atomic optics" focussing technique in our ultra-cold spin-polarized atomic hydrogen jet target. Assuming specular reflection and a point source, a parabolic mirror should form a parallel beam of monochromatic atomic hydrogen. Such a mirror could significantly increase the beam available for focussing by a sextupole magnet. Used quasi parabolic mirror has a special shape taken into account that the magnet field gradient accelerates atoms and bent them trajectory before reflection. The copper four-coned approximation mirror was installed with its focus at the $5 \mathrm{~mm}$ diameter exit aperture of the atomic hydrogen stabilization cell (see Fig. 2). 


\section{Helium Film Coated Quasi-Parabolic Mirror}

We first made baseline measurements with no mirror. The maximum signal was observed at the largest central solenoid field of 7.3 T (see Fig. 3); this gave the largest gradient which increased both the electron spin separation inside the cell and the solenoid fucusing outside.
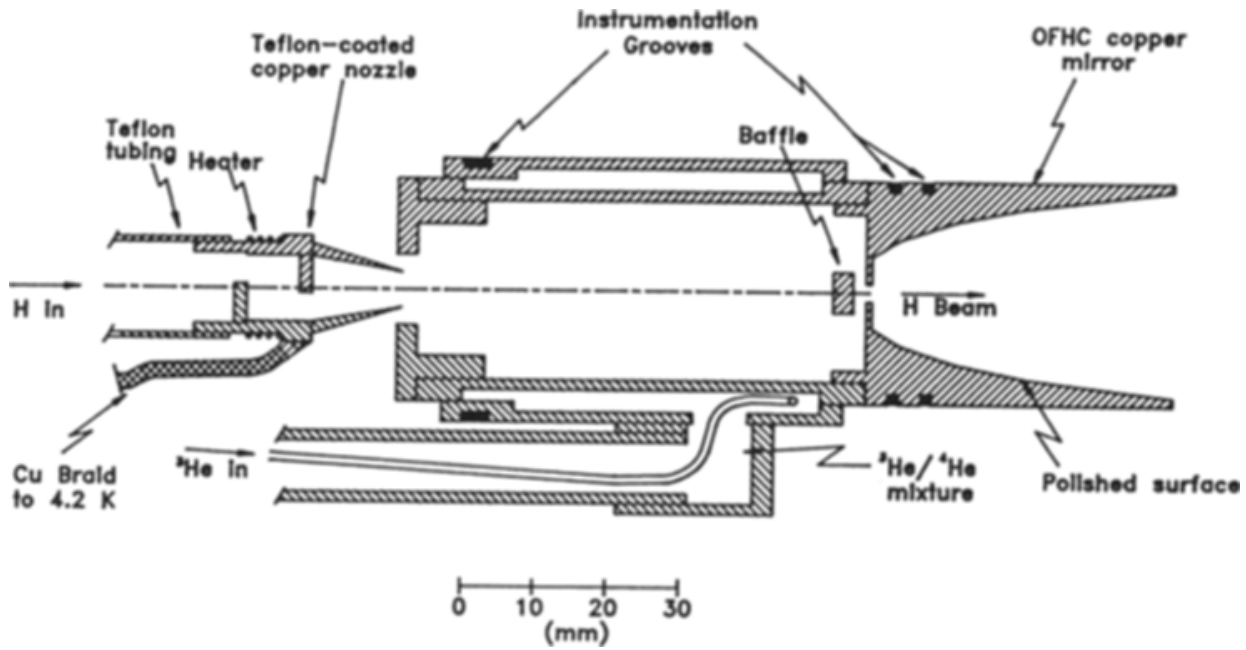

FIG. 2. Schematic diagram of the stabilization cell and mirror. The Tefloncoated copper nozzle is also shown.

The measured CT signal for the mirror polished with a 1,000 nm abrasive versus the solenoid magnetic field are plotted in Fig. 3. At a field of $7.5 \mathrm{~T}$ the four-coned mirror's CT signal was $2.4 \cdot 10^{15} \mathrm{H} \mathrm{s}^{-1}$, this was about 4.8 times larger than the extrapolation to $7.5 \mathrm{~T}$ of the signal with no mirror.

For formation of a low divergence beam using the specular reflection, the mirror surface should be at least no rougher than the thickness of the helium film on the surface, which is typically about $10 \mathrm{~nm}$. Therefore, we repolished the mirror surface with a $50 \mathrm{~nm}$, alumina suspension; this significantly reduced the surface roughness. With this repolished surface the measured peak CT intensity at $8 \mathrm{~T}$ increased to $3.7 \cdot 10^{15} \mathrm{H} \mathrm{s}^{-1}$. Comparison with the no mirror baseline extrapolation to $8 \mathrm{~T}$ indicates that the repolished mirror increased the measured beam intensity by a factor of about 7.5 . 


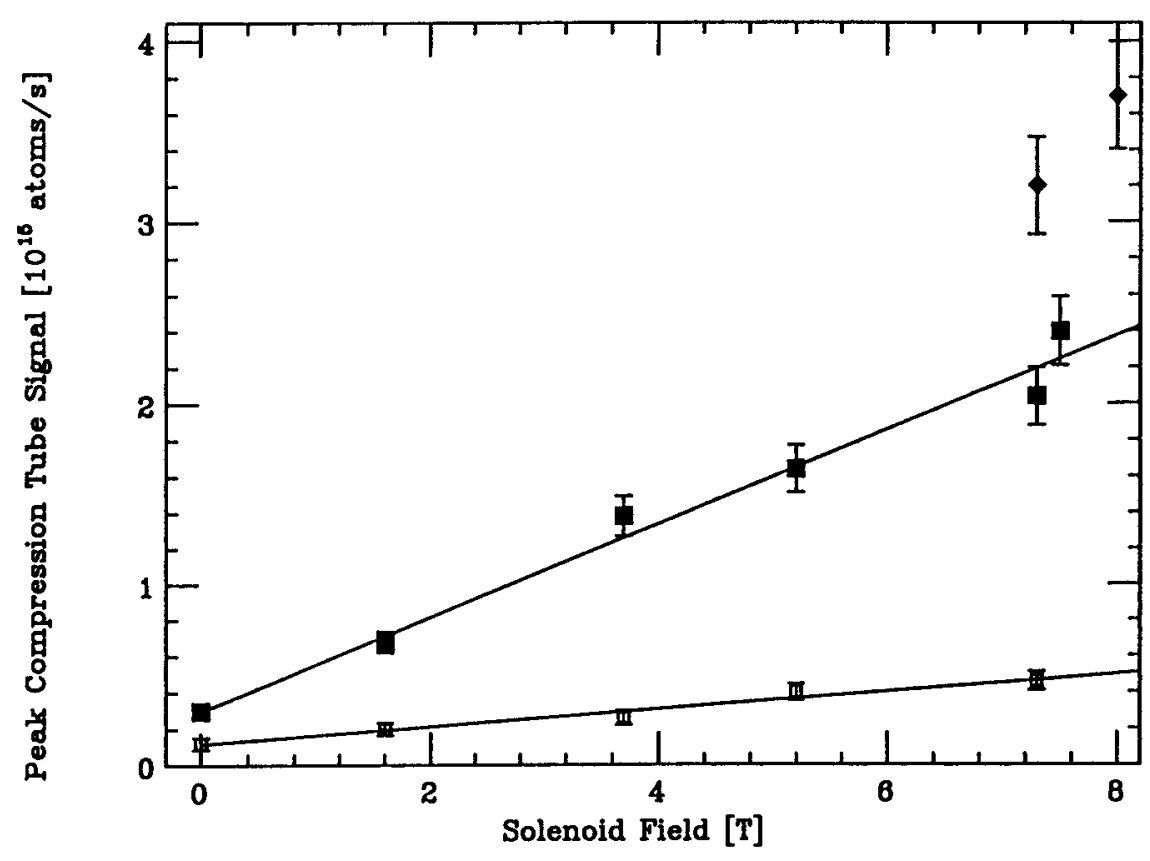

FIG. 3. The peak compression tube signal at each optimal sextupole current is plotted versus the solenoid field. The open squares are with no mirror; the black squares are the four-coned mirror with a rougher surface; the black diamonds are with the repolished four-coned mirror. The curves are linear least square fits to the data.

In summary, this work demonstrates the possibility of forming an ultra-cold atomic hydrogen beam by using a polished mirror coated with a superfluid ${ }^{4} \mathrm{He}$ film. We plan to design and test a field gradient mirror for our new $12 \mathrm{~T}$ solenoid.

\section{ACKNOWLEDGEMENTS}

This work was supported by a research grant from the U.S. Department of Energy.

\section{REFERENCES}

* Permanent address: Joint Institute for Nuclear Research, Dubna, Russia.

1. J. J. Berkhout et al., Phys. Rev. Lett. 63, 1689 (1989).

2. T. Roser et al., Nucl. Instrum. Methods A301, 42 (1991). 